\title{
An Architecture for Using Commodity Devices and Smart Phones in Health Systems
}

\author{
Geir Horn ${ }^{1}$, Frank Eliassen ${ }^{1}$, Amir Taherkordi ${ }^{1}$, Salvatore Venticinque ${ }^{2}$, Beniamino \\ Di Martino ${ }^{2}$, Monika Bücher ${ }^{3}$, and Lisa Wood ${ }^{3}$ \\ 1 University of Oslo, P. O. Box 1080, 0316 Oslo, Norway \\ geir.horn@mn.uio.no, frankeifi.uio.no, amirhost@ifi.uio.no \\ 2 Seconda Università degli Studi di Napoli, Via Antonio Vivaldi, 43, 81100 Caserta CE, Italy \\ salvatore.venticinque@unina2.it, bedimarteunina.it \\ 3 Lancaster University, Bailrigg, Lancaster LA1 4YW, United Kingdom \\ m.buscher@lancaster.ac.uk, l.a.wood@lancaster.ac.uk
}

\begin{abstract}
The potential of patient-centred care and a connected eHealth ecosystem can be developed through socially responsible innovative architectures. The purpose of this paper is to define key innovation needs. This is achieved through conceptual development of an architecture for common information spaces with emergent end-user applications by supporting intelligent processing of measurements, data and services at the Internet of Things (IoT) integration level. The scope is conceptual definition, and results include descriptions of social, legal and ethical requirements, an architecture, services and connectivity infrastructures for consumer-oriented healthcare systems linking co-existing healthcare systems and consumer devices. We conclude with recommendations based on an analysis of research challenges related to how to process the data securely and anonymously and how to interconnect participants and services with different standards and interaction protocols, and devices with heterogeneous hardware and software configurations.
\end{abstract}

Key words: Healthcare bus, IoT, Architecture

\section{Introduction}

Europe has the second highest smart phone penetration in the world ${ }^{1}$, and for many European citizens, this creates opportunities to interact with public services and each other in new ways. An emerging big trend ${ }^{2}$ in portable consumer electronics is wear$a b l e s^{3}$, and there are already abundance of devices available to monitor a person's biodata and daily activities. However, the existing IoT solutions for healthcare are often vertically-oriented closed systems, making it impossible to produce consumer-oriented applications to generate a strong cooperation between the plethora of consumer devices

\footnotetext{
${ }^{1}$ http://www.emarketer.com/Article/Worldwide-Smartphone-Usage-Grow-25- 
like smart phones and wearables, medical devices, patient monitoring systems, hospital information software, and other external services.

If the key European Commission strategic priority ${ }^{4}$ of connecting healthcare systems is to be met, a novel architecture is needed for achieving wider interoperability of eHealth services in ways that are not currently possible. In particular, this architecture needs to consider the integration of both data, services, and things. While we acknowledge that most of existing solutions provide a good basis for integration of IoT systems $[2,3,6]$, they fail to provide a unified platform for integrated data, services, and things. This novel platform needs to accommodate the current de facto standards and patterns for integration, and dynamically support future and emergent integration needs and protocols. It will enable applications like an "heart desease monitoring app" on smart phones that, when connected to appropriate body sensors (e.g., blood pressure, heart rate, GPS), can automatically report alarming conditions directly to the hospital's information system. this in turn may then take further actions like checking the person's medical record, and make a reservation for a full check. The assumed beneficiaries of this integration strategy are seen as three fold:

Patients who will experience enhanced safety of treatments received, delivery of care at the point of need, integrated care and safe treatment abroad, e.g. in an emergency situation in another EU Member State;

Healthcare professionals who will see improved quality and safety of care through strengthened coordination; up-to-date patient status information and real-life patient monitoring and evidence-based clinical guidelines to support decision-making procedures;

Other Users (e.g. insurers, employers, service providers) will have access to interoperable systems resulting in significantly lower implementation and integration costs.

Reconciling informational self-determination and visions of eHealth is an ambitious goal that raises challenges for technical interoperability, sociotechnical ordering and the management of data for diverse parties with diverse interests. It is happening already: Health and life insurers are beginning to encourage their customers to use self-tracking devices and apps to collect personal health and fitness data and upload these data to their platforms in the interests of gaining "rewards" or being offered lower premiums [1]. However, technical connectivity and data sharing does not equate to interoperability. To successfully design for collaborative work in integrated healthcare, "common information spaces" are needed [7]. The proposed architecture is therefore an integration solution, responsibly designed within an ethically, legally and socially responsible framework, for isolated healthcare information system silos, consumer devices, and fragmented IoT architectures.

\section{Socially Responsible Common Information Spaces}

Designing Common Information Spaces that are not just data "oceans" but that bring together mechanisms to support discretionary disclosure, mutual intelligibility and col-

\footnotetext{
4 http://ec.europa.eu/digital-agenda/interoperability-standardisation-

connecting-ehealth-services
} 
laborative reasoning undoubtedly requires a transformation of healthcare structures. Instead of conceiving patients merely as "experiencers" of health problems and health professionals as expert diagnostic agents, this reconfiguration requires engagement and the transfer of authority to citizens to manage their health and care [9]. As such, and in order for integrated healthcare to succeed, we need to acknowledge the positively disruptive potential of innovation. New technologies can lead to productive new arrangements of responsibilities between providers, patients and consumers. This has the potential to have significant implications for the practice of medicine, medical authority, the delivery of healthcare and public health work and the doctor-patient relationship ${ }^{5}$.

Achieving this potential has to acknowledge and account for the reasons why people track their data, which may not be to monitor their health. People track bio-data for entertainment and social reasons, creating "data spectacles" [8], performances of selfhood and a mode of creative expression. Bringing these diverse forms of data together creates challenges for reliability, intelligibiltiy and authority.

A further social challenge to the integration of these services comes from the impossibility of app designers, and those who develop digital technologies for medical and health related purposes, to recognise the social and cultural differences that may influence how people interact with devices. Beliefs about health and illness vary from culture to culture, as do responses to health technologies and it is impossible predict how these systems may be appropriated by users [1].

Furthermore, the transference of one system into another also raises legal questions regarding the use of data and the regulation of the system. Apps that make more sophisticated calculations and interpret data for therapeutic or diagnostic purposes are more likely to be considered as medical devices and thus subject to extensive legal controls and approvals. Design should consider how new technologies may support diversity, balance concerns with quality with a desire for economy and efficiency, and ameliorate negative unintended consequences. A socially aware, socially responsible design process, can allow innovation to be sensitive to such social, legal, and ethical issues.

\section{Architecture}

The above discussion illustrates that the architecture must be based on some salient features:

1. The connectivity should be ubiquitous;

2. Application modules must be able to connect to each location transparently and should support state of the art privacy preserving techniques, such as role-based access management, accountable data access and Privacy Enabling Transparent Systems (PETS);

3. New and existing healthcare devices must be seamlessly interfaced and integrated; and

4. The applications should be component based software-only applications.

\footnotetext{
5 https://simplysociology . wordpress.com/2015/03/22/managing-and-

materialising-data-as-part-of-self-tracking/
} 
Fundamentally, we propose the architecture to be structured as an Enterprise Software Bus (ESB). The main purpose of an ESB is to ensure that components can connect with each other and exchange messages. An ESB can also provide component directories, monitoring, security aspects, and actual application composition from semantic descriptions of available components. To this end, we propose a layered architectural approach as illustrated in Figure 1.

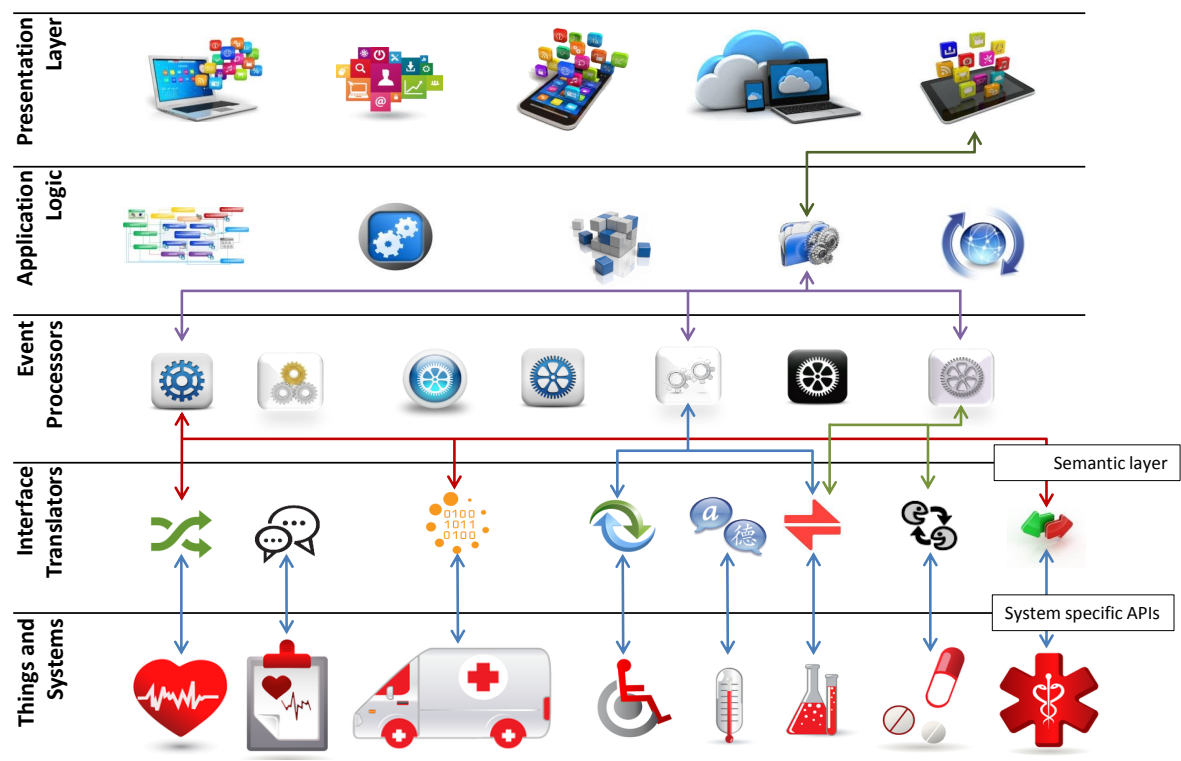

Fig. 1. The layered approach to patient oriented application integration proposed for IoT based healthcare systems.

At the lowest layer, things and systems represent connected devices, sensors and systems already used inside hospitals, in mobile environments, at laboratories, at home or outdoors for measuring health related parameters. At the next layer up, interface translators are necessary to allow access to a myriad of diverse devices. Importantly, all devices and sub-systems have interfaces that are often proprietary and not standardized for certified healthcare appliances. To allow such devices and sub-systems to be integrated in patient oriented applications, it is necessary to translate their APIs into a homogeneous or interoperable form. This translation will be specific to the protocol used by every device, and in principle there will be one translator for each device to interface. Such translators will typically, in the future, be provided by the device manufacturers, or third parties will be able to develop and sell them to system integrators developing applications accessing such devices.

In order to better support interoperability of devices, we believe the translated APIs need to be semantically enriched using a health device semantic representation based 
on the available ontologies for the health domain ${ }^{6}$. Despite the multitude of available ontologies, the integration of these heterogeneous sources of patient information and various healthcare domains is non-trivial. Furthermore, it is necessary to integrate the existing ontologies by complementing them with additional information able to support the automatic integration and interoperability between health devices, personal devices and useful software systems. Thus, the semantic layer includes the abstract description of the functionalities offered and the data exchanged by devices and software systems by means of a semantic model realized through ontologies. Importantly, in order to ease the task of integration there is a need to investigate techniques to support the automatic construction of interface translators by matchmaking the device API with the abstract representation provided by the semantic layer.

Event processors connect to one or more of the translators through their semantic interfaces. They are understood as generalised processors that filter and analyse information according to specific objectives, and event processors can take input from other event processors' output. Thus, an application can employ a hierarchy of event processors. Event processors can be simple aggregation oriented filters, like calculating the mean value of a sensor's outputs, or focused on complex event detection by combining information from several sources. In abstract terms, they serve two main purposes. Firstly, they should ensure that the data from the monitored systems and devices are kept private, and event processors should therefore run as close to the sensor as possible, given the processors' requirements for memory and processing capabilities. Secondly, they should help to reduce the amount of information sent over the network.

Application Logic is the core functionality of the patient centric application based on the output from the event processors. Because patients can be mobile, applications should be adaptive and reactive to the user's current context. Network mobility and transparent handoffs and handover must be supported. The architecture must combine established software concepts like introspection and reflection with context information and novel ideas on stochastic learning to allow dynamic distributed reactive run-time reconfiguration of the application $[4,5]$. The presentation layer is where the application interacts with the diverse users or the healthcare personnel. This is very application focused, and the user interaction is essential for the end-user acceptance of an application and its value.

\section{Services}

Service integration will be driven by semantic-based, predefined, application patterns describing a set of software components and their orchestration needed to implement the application's functionality, or to fulfil an objective. An automatic inference process to select the best suited application pattern from the catalogue is needed. Our proposal is that it must be based on a mix of predefined rules and utility function based adaptation techniques, combined with fuzzy goal representation and reinforcement learning [5]. Once the application pattern has been selected, the application must be adapted to this new pattern in a safe way.

\footnotetext{
${ }^{6}$ http://www.openclinical.org/ontologies.html
} 
A multi-agent system (MAS) will implement a monitoring system [10]. A software platform executing on embedded and computing nodes will allow for hosting agents to collect, elaborate and exchange performance information for the assessment of service levels. It will provide an execution environment for agents, communication facilities and mobility mechanisms. In fact, mobility will be used to dispatch specialized agents for estimating those parameters that are relevant to continuously meet application requirements, dependent upon the user's health information to be controlled. A MAS is chosen since agents will be able to execute autonomously even during connection failures, granting real-time reaction and continuous checking of operational parameters, such as availability, precision, time resolution, session persistence among sub-systems, availability of a resource and of the network, quality of the interconnection service, etc.

Event processors can inter-operate on the semantic layer, realized through ontologies, that includes the abstract description of the functionalities offered and the data exchanged by devices and software systems. Interface translators must be used to allow access to a myriad of diverse devices. In fact, all devices and sub-systems have interfaces, although they are often proprietary and not standardised for certified healthcare applications.

\section{Connectivity}

We propose to build the architecture on top of the Data Distribution Service (DDS ${ }^{7}$ ) standard from the Object Management Group $\left(\mathrm{OMG}^{8}\right)$, for which there is an open source implementation ${ }^{9}$ that can serve as the basis for the proposed healthcare bus. The DDS standard was driven by the needs for a ubiquitous data bus for mission critical systems of systems within the aerospace and defence domains, and evolved to encompass on-the-wire interoperability. DDS offers a real-time publish-subscribe model on top of the Internet User Datagram Protocol (UDP) transport protocol, and it is based on OMG's Interface Description Language (IDL ${ }^{10}$ ) specification language, which provides a way to define interfaces independent of the underlying platform, the programming language, or the operating system.

\section{Conclusion}

This brief paper has analysed some of the issues in creating a common information space to integrate consumer electronic devices into professional healthcare, and outlined an ethically, legally and socially responsible architecture that may foster ecosystems of user centric healthcare applications. The necesary technological building blocks to realise this architecture have been identified.

\footnotetext{
7 http://portals.omg.org/dds/content/page/dds-specifications

8 http: //www.omg.org/

9 http://www.opendds.org/index.html

10 http://www.omg.org/gettingstarted/omg_idl.htm
} 


\section{References}

1. D. Lupton. Apps as artefacts: Towards a critical perspective on mobile health and medical apps. Societies, 4:606-622, 2014.

2. Dominique Guinard, V. Trifa, S. Karnouskos, P. Spiess, and D. Savio. Interacting with the SOA-Based Internet of Things: Discovery, Query, Selection, and On-Demand Provisioning of Web Services. IEEE Transactions on Services Computing, 3(3):223-235, July 2010.

3. Dominique Guinard, Vlad Trifa, Friedemann Mattern, and Erik Wilde. From the Internet of Things to the Web of Things: Resource-oriented Architecture and Best Practices. In Dieter Uckelmann, Mark Harrison, and Florian Michahelles, editors, Architecting the Internet of Things, pages 97-129. Springer Berlin Heidelberg, 2011.

4. S. Hallsteinsen, K. Geihs, N. Paspallis, F. Eliassen, G. Horn, J. Lorenzo, A. Mamelli, and G. A. Papadopoulos. A development framework and methodology for self-adapting applications in ubiquitous computing environments. J. Syst. Softw., 85(12), 2012.

5. G. Horn and B. J. Oommen. Solving multiconstraint assignment problems using learning automata. Trans. Sys. Man Cyber. Part B, 40(1), 2010.

6. John Soldatos, Nikos Kefalakis, Manfred Hauswirth, Martin Serrano, Jean-Paul Calbimonte, Mehdi Riahi, Karl Aberer, Prem Prakash Jayaraman, Arkady Zaslavsky, Ivana Podnar Žarko, Lea Skorin-Kapov, and Reinhard Herzog. OpenIoT: Open Source Internet-of-Things in the Cloud. In Ivana Podnar Žarko, Krešimir Pripužić, and Martin Serrano, editors, Interoperability and Open-Source Solutions for the Internet of Things: Proceedings of the International Workshop of the FP7 OpenIoT Project (SoftCOM 2014), Lecture Notes in Computer Science 9001, pages 13-25, Conference Location: Split, Croatia, Sept. 2014. Springer International Publishing.

7. L. Bannon and S. Bødker. Constructing common information spaces. In J. Hughes, editor, Proceedings of the Fifth European Conference on Computer Supported Cooperative Work, pages 81-96. Kluwer, 1997.

8. M. Gregg. Inside the data spectacle. Telev. New Media, 16:37-51, 2015.

9. N. Saranummi. In the spotlight: Health information systems. IEEE Rev. Biomed. Eng., 4:17-19, 2011.

10. M. Rak, S. Venticinque, T. Máhr, G. Echevarria, and G. Esnal. Cloud application monitoring: The mosaic approach. pages 758-763, 2011. 